\title{
FLASH Syndrome: Tapping into the Root of Chronic Illness
}

\author{
Michael Raymond Binder \\ North Shore University Health System, Highland Park Hospital, Highland Park, Illinois, USA
}

Email address:

mbinder@drmichaelbinder.com

\section{To cite this article:}

Michael Raymond Binder. FLASH Syndrome: Tapping into the Root of Chronic Illness. American Journal of Clinical and Experimental Medicine. Vol. 8, No. 6, 2020, pp. 95-103. doi: 10.11648/j.ajcem.20200806.11

Received: November 3, 2020; Accepted: November 13, 2020; Published: November 23, 2020

\begin{abstract}
It has now been established that persons with higher resting heart rates are at increased risk of morbidity and mortality from a plethora of mental and physical illnesses. However, the mechanism underlying the predictive value of this core vital sign remains obscure. This seminal report will integrate clinical, neuropsychological, physiological, and genetic evidence to assert that an inherent hyperexcitability of the neurological system is at the heart of the connection between resting heart rate and disease. Hypothetically, neuronal hyperexcitability can cause multiple circuits in the brain to overfire, including cognitive circuits, limbic circuits, and autonomic circuits, thereby dysregulating the associated systems of the body and allowing the aberration to be detected through standard vital signs and related measures of autonomic activity. Because the cognitive-emotional system is exquisitely sensitive to neuronal excitation, the aberration could also manifest as psychiatric symptomatology, thus suggesting that psychiatric symptoms may be the first subjective markers of the abnormality. Based on the well-recognized link between mental illness, autonomic dysregulation, and systemic disease together with mounting evidence that the related illnesses are associated with gene variants whose protein products fail to adequately regulate the firing of neurons, the vulnerability trait could aptly be called Familial Limbic Autonomic System Hyperexcitability or "FLASH." Because the trait appears to be so common, its effects so pervasive, and its expression so modifiable, its identification is of critical importance to every medical specialty. FLASH could give clinicians the first comprehensive biological target through which to treat and prevent a plethora of mental, emotional, and physical illnesses.
\end{abstract}

Keywords: Prognostic Indicators, Heart Rate, Heart Rate Variability, Heart Rate Recovery, Respiratory Rate, Neuronal Hyperexcitability, Molecular Genetics, Ion Channelopathies

\section{Introduction}

It is now clear that persons with higher resting heart rates (RHRs) are at significantly increased risk of developing a wide range of physical illnesses including high blood pressure [1-4], type-2 diabetes [5-7], cardiovascular disease [8-13], cerebrovascular disease [14-16], kidney disease [17], cancer [16, 18, 19], dementia [20], and sudden death [21]. Higher RHRs have also been associated with a wide range of mental and emotional illnesses, including major depressive disorder, generalized anxiety disorder, panic disorder, obsessive-compulsive disorder, post-traumatic stress disorder, and psychotic disorders [22-26]. Although less well-studied in relation to RHR, psychiatric disorders have long been associated with autonomic dysregulation [27-29], and most persons with severe mental illness die of the same kinds of diseases as the general population [27, 30]. However, for unclear reasons, they die at a much earlier age [27, 30].

This seminal report will examine the connection between RHR and vulnerability to illness. From there, it will posit the underlying abnormality, trace its origin, and discuss the means by which the abnormality can be identified and modified so as to improve the long-term health and wellbeing of those affected by it.

\section{Examining the Mechanism of the Connection}

The RHRs that have been linked to an increased vulnerability to illness are not necessarily outside the accepted range of "normal" but rather on the upper end of normal, with most studies reporting a significant increase in disease risk with RHRs above 70 beats/min [2-22]. Other measures that have been linked to an increased vulnerability 
to illness include resting respiratory rate (RRR) [31-33], resting blood pressure (RBP) [22, 34, 35], blood pressure variability $[34,36]$, heart rate variability $[4,36-38]$, and heart rate recovery $[21,39]$. The increased risk of morbidly and mortality associated with these indices can be more than twofold $[21,31,37,40]$. This is particularly concerning because it includes the world's leading cause of death-heart disease. What's more, the actual risk of illness, as will be discussed, is often much greater than statistically indicated, thus making it all the more important to get to the heart of the connection between constitutionally-elevated vital signs and disease.

Because all three vital signs-RHR, RRR, and RBP - are regulated by the autonomic nervous system (ANS), all three (and their correlates) reflect on the activity of the ANS. The ANS has two divisions: the sympathetic division, which is activated when there is a call to action, and the parasympathetic division, which regulates the digestive and recuperative functions of the body. When there is a call to action, the sympathetic nervous system drives an increase in heart rate, heart contractility, and respiratory rate in anticipation of an increased need for oxygen. When the job is done, sympathetic activity decreases, and parasympathetic activity increases [41].

Based on these physiological dynamics, a chronic elevation in the ratio of sympathetic-to-parasympathetic activity, as indicated by higher RHRs and other indices of autonomic function, would maintain the body in a heightened catabolic state - more breaking down than building up. This would naturally tend to accelerate the aging process and hasten the onset and progression of diseases that ultimately lead to the end of life $[42,43]$. The pressing question is: what causes the chronic dysregulation of the ANS?

\section{Shared Effect on Mental Health}

An important clue to what persistently dysregulates the ANS is the increased risk of psychiatric disorders that is associated with constitutionally-elevated vital signs [22, 44]. Although the pathophysiology of mental illness remains unclear, an emerging hypothesis contends that psychiatric symptoms are driven by pathological hyperactivity in symptom-related circuits in the brain. According to the Multi-Circuit Neuronal Hyperexcitability (MCNH) hypothesis of psychiatric disorders, persistent firing in anxiety circuits causes persistent feelings of anxiety; persistent firing in depressive circuits causes persistent feelings of depression; persistent firing in cognitive circuits causes ruminative and obsessive thoughts; etc...[45]. That is not to say that the brain as a whole is pathologically hyperactive. Rather, the pathological hyperactivity is thought to occur in the brain's microcircuitry $[45,46]$, with hotspots of activity that either remain focal or migrate from one circuit loop to another like a wandering tornado [45]. The theoretical means by which this occurs is that the locus of hyperactivity, like a short-circuit in a wired electrical system, inappropriately fuels activity in circuits that would remain less active were they not themselves hyperexcitable [45, 47].
In addition to increasing activity in the cognitive-emotional system, neuronal hyperexcitability would tend to increase activity in the sensory system, the motor system, the endocrine system, and other systems of the body, including the ANS [42, 45, 47-50]. These systems must be tightly regulated to maintain optimal health, and any lack thereof would heighten the risk of both acute symptoms and longterm illness. Thus, in addition to explaining both the production of symptoms and the migration of symptoms in psychiatric disorders, an inherent hyperexcitability of the neurological system could explain the various functional symptoms that are associated with psychiatric disorders; it could explain the chronic dysregulation of the ANS that is associated with psychiatric disorders; and it could explain the early onset of disease that is associated with psychiatric disorders [27, 30]. Such broad explanatory power would hypothetically place neuronal hyperexcitability at the heart of the connection between constitutionally-elevated vital signs and chronic disease.

The excitability of neurons is governed by electrical gradients across neuronal membranes and the flow of ions through their respective channels. Consistent with the MCNH hypothesis, drugs that influence these channels - specifically benzodiazepine and non-benzodiazepine anticonvulsantshave been used for decades in the treatment of psychiatric disorders, and the anticonvulsant-like drug lithium was the first to be used [51-53]. Then again, reducing excitation in overactive cognitive and dysphoria-related circuits is not, from the perspective of the MCNH hypothesis, the only means by which psychiatric symptoms could be reduced. Symptoms could also be reduced by increasing excitation in pleasure-related circuits (as with tricyclic antidepressants, serotonin-norepinephrine reuptake inhibitors, and psychostimulants) and by modulating activity in these circuits (as with selective serotonin reuptake inhibitors) [46, 54-56]. Note, however, that any associated increase in the excitatory/inhibitory balance would tend to further dysregulate the system, thus increasing the risk of aberrant circuit induction. This is demonstrated by the potential of these drugs to cause paradoxical effects, rapid cycling, and conversion to mania [57-61]. It is also demonstrated by their overall tendency, even when used successfully to treat anxiety and depression, to adversely affect RHR, heart rate variability, and other novel markers of disease risk [62-69]. Although a complete discussion of the MCNH hypothesis is beyond the scope of this article, it should be noted that there is strong biological, pharmacological, psychophysiological, neuropsychiatric, radiologic, medical, experimental, electrophysiological, observational, behavioral, and explanatory evidence for the hypothesis [45, 47].

Persons with psychiatric disorders are quick to react [70] and slow to recover [43] from stressful events. Even in the absence of specific stressors, they tend to be stressed by abstract worries and fears [43, 71]. During that time, the sympathetic nervous system, which is tightly integrated with the cognitive-emotional system [72], would be activated beyond its already elevated basal activity $[42,43]$. It would 
also be activated more steeply [70] and more robustly than normal (Figure 1A) [42, 43, 70]. In addition, the prolonged recovery time would increase the likelihood that the autonomic responses to repeated stressors would coalesce or even summate (Figure 1B) [42]. Were this to occur, it would create a chronic allosteric load that would markedly accelerate the aging process and hasten the onset of illness $[42,43,70]$. In addition to amplifying stress, an inherent hyperexcitability of the neurological system could, through maladaptive behavior, drive the development of the stressors themselves, thus orchestrating a vicious cycle of pathological stress and reasons to be stressed [42]. This neuropsychological dynamic would again place neuronal hyperexcitability at the heart of the connection between constitutionally-elevated vital signs and disease.
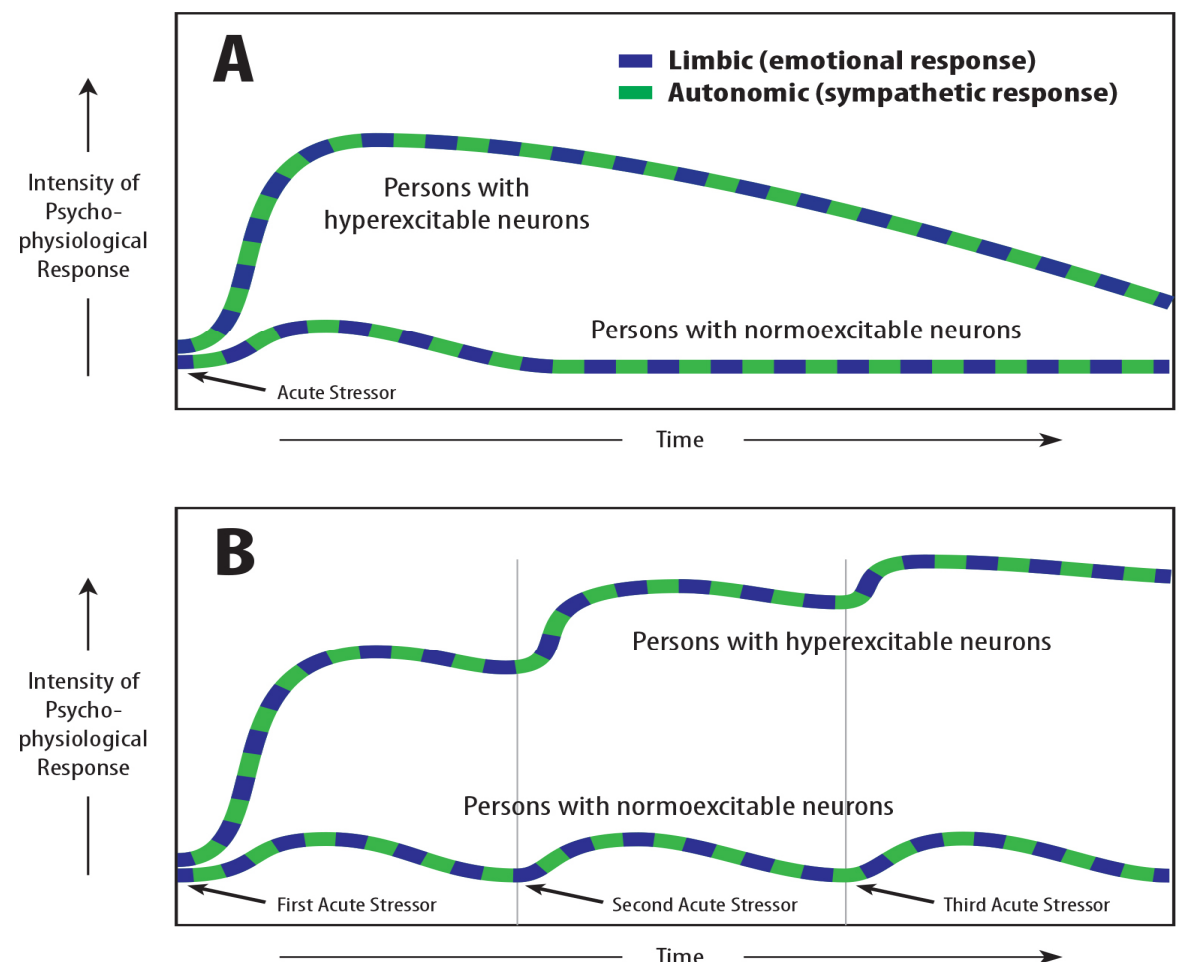

Figure 1. Schematic illustration of the stress-response curves in persons with hyperexcitable neurons compared to those with normoexcitable neurons A) when there is a single stressor; B) when there is a rapid series of stressors. Note: color variegation depicts the limbic and autonomic responses tracking together.

Figure 1 schematically illustrates how dramatically the response to stress can be amplified by hyperexcitable neurons: A) in response to a single stressor; B) in response to a rapid series of stressors. Hypothetically, this pathological amplification of neural signaling is what increases an affected person's risk of developing any illness that could be brought on by severe or persistent stress.

\section{The Many Faces of Neuronal Hyperexcitability}

That leads to another question: if neuronal hyperexcitability were at the heart of the connection between vital signs and disease, why is it that only some persons with constitutionally-elevated vital signs develop psychiatric disorders?

The answer lies in the same set of factors that would determine which, among many possible psychiatric disorders, if any, a person with hyperexcitable neurons would develop; namely, the complex dynamic between trait severity, brain architecture, developmental factors, situational factors, and the coping skills of the individual $[42,73,74]$. The idea of a shared diathesis with variable expression is supported by the high degree of genetic correlation among many psychiatric disorders and by the high correlation of the personality trait of neuroticism with almost every psychiatric disorder [71]. Also, it is not uncommon for various combinations of psychiatric symptoms to come and go or wax and wane in severity over the course of an affected person's lifetime. Although the severity of symptoms is largely determined by one's perceived level of stress, the constellation of symptoms that will develop during a given psychiatric episode is more difficult to predict, even in the same individual [73, 74]. What is predictable is that symptoms will tend to resolve or completely disappear during low-stress periods [70, 73]. Hypothetically, the reduction in stress reduces intrapsychic tension, thus allowing the pathological hyperactivity in the hyperexcitable brain to dissipate $[45,47]$.

Then again, because the excitation-to-inhibition balance in the hyperexcitable brain would be tonically elevated, the dissipation of pathological hyperactivity consequent to stress reduction would not necessary leave an affected person symptom-free. Any persistent symptomatology would, however, be more likely to fall short of one of the currently accepted DSM-5 psychiatric disorders [43]. Such 
subsyndromal symptomatology could include excessive worry, excessive fear, obsessive thoughts, moodiness, irritability, impatience, inattentiveness, and trouble sleeping. Although generally perceived as extremes of normal, these symptoms, like upper-end-of-normal vital signs, are both associated with psychiatric disorders $[44,71]$ and predictive of their development [22, 43, 74]. Also, many of these symptoms, having been variably described as "neurotic" [22], "ruminative" [43], "Type A" [75], or "Type D" [76] personality, have been independently linked to autonomic dysregulation and the development of cardiovascular disease $[43,75,76]$. Other seemingly non-psychiatric ways that neuronal hyperexcitability could manifest include hypersensitivity to sound or light, recurrent headaches, fibromyalgia, irritable bowel, chronic pain, and somatoform disorders [45, 48]. There are also some persons who, for cultural reasons or to avoid social stigma, unconsciously "convert" their emotional pain into physical symptoms. Finally, some persons may mask, distort, or temporarily quell their symptoms through the use of alcohol and other psychoactive substances. Notably, the two most commonly used substances - alcohol and cannabis - are potent anticonvulsants, an observation that could explain the high frequency with which they are used to self-medicate. For all of these reasons, the absence of overt psychiatric symptoms would not necessarily mean that a person did not have hyperexcitable neurons.

\section{Probing the Cause of Neuronal Hyperexcitability}

Although there are many state-dependent factors that could cause the neurological system to become pathologically hyperexcitable, such as metabolic disturbances, inflammatory states, and stimulant-type drugs, these would not explain the hyperexcitability that is hypothetically at the root of constitutionally-elevated vital signs. The cause of the abnormality would have to be something more fundamental and more deeply rooted than that.

An important clue to what that abnormality might be comes from gene association studies for common psychiatric, neurologic, and general medical conditions. The most robust and consistent outcome of these studies has been the identification of gene variants whose protein products fail to adequately regulate the excitability of neurons [71, 77-89]. Consistent with the wide distribution of neurons (and other excitable cells) throughout the body, ion channelopathies have been linked to a plethora of illnesses including anxiety $[74,83,87]$, depression [74, 79, 80, 87], bipolar disorder [74, 77-81, 87], schizophrenia [74, 79, 84-87], autism [74], epilepsy [78, 88, 89], migraine headache [88], irritable bowel syndrome [88], diabetes mellitus [88], high blood pressure [88], cardiac conduction abnormalities [88, 89], renal abnormalities [88], asthma [88], cystic fibrosis [88], peripheral pain syndromes $[89,90]$, and cancer $[88]$. That the genetic underpinnings of these disorders would cause a hyperexcitability of the neurological system would once again place neuronal hyperexcitability at the heart of the connection between constitutionally-elevated vital signs and disease. It would also reveal the probable source of the abnormality.

\section{How to Identify the Vulnerability Trait}

In contrast to the gradual erosive effects that neuronal hyperexcitability would have on physical health, its effects on mental health would be more readily apparent due to the exquisite sensitivity of the cognitive-emotional system to neuronal excitation [46]. Every time an emotional stressor were experienced, the hyperexcitable brain would abnormally amplify the stress, thus increasing the potential for psychiatric and related functional symptoms to emerge [42, 43]. Moreover, the severity of the symptoms and magnitude of the stress-induced adverse effects on general health would be proportionate to the severity of the neuronal hyperexcitability trait. That could explain why persons with the most severe psychiatric symptoms have the shortest lifespans $[27,30]$. It could also explain why they die of the same kinds of illnesses as the general population [27, 30]. From this perspective, psychiatric symptoms could be reconceptualized as the first subjective markers of a chronic dysregulation of the ANS and, hypothetically, of an underlying hyperexcitability of the neurological system. Then again, as previously discussed, the behavior of hyperexcitable neurons tends to normalize during low-stress periods, thus allowing psychiatric symptoms to remit during those times. In addition, social stigma and lack of clarity about the cause of mental illness lead many persons to rationalize, self-medicate, or deny their symptoms even when they are overtly present. These factors could potentially reduce the reliability of psychiatric symptoms as markers of neuronal hyperexcitability. However, the well-established connection between RHR, autonomic dysregulation, and the development a wide range of mental and physical illnesses strongly suggests that RHR continues to flag the trait of neuronal hyperexcitability whether or not a person is symptomatic. Moreover, in all of the RRR studies, which include RRR in relation to either autonomic function, current illness, or risk of illness, the relationship was not linear but rather dichotomized, with RRRs above 15 breaths/min being independently associated with an elevation in sympathetic drive [49], emotional disorders [44], and all-cause morbidity and mortality [31-33]. This dichotomization suggests that there is a gene-related difference between the two groups and, hence, that RRR may be a more reliable marker of the trait of neuronal hyperexcitability and its underlying channelopathies. Although RHRs likewise showed some tendency to dichotomize (mainly above 75 beats/min) $[4,9,11,15,18,19$, 21] the pattern with RRRs was more distinct, a finding that may be explained by the superior long-term stability of RRR in comparison to RHR and other novel markers of disease risk [91].

Based on evidence that neuronal hyperexcitability has a 
genetic origin and would most readily be expressed clinically through perturbations in the limbic and autonomic nervous systems, the trait could aptly be called Familial Limbic Autonomic System Hyperexcitability, or FLASH. The acronym "FLASH" fittingly recapitulates the fundamental abnormality that it describes; namely, a hyperexcitability of the neurological system that causes circuit-specific electrical storms in the brain. What would distinguish FLASH storms from seizure activity is that in FLASH the involved neurons would be fewer in number, circuit-specific, and synchronous rather than hypersynchronous in their activity [45, 47]. Consequently, they would not induce the large magnetic fields that are associated with seizure activity [47]. This conceptualization is supported by multiple lines of evidence including the long-recognized connection between seizure disorders and psychiatric disorders [47], the well-recognized benefits of anticonvulsant drugs in psychiatry [92, 93], and the ability of anticonvulsant drugs to improve heart rate variability [94], a well-recognized index of autonomic activity $[37,62,63]$.

\section{Discussion}

The evidence base for the prognostic potential of RHR and other indices of autonomic activity is exploding, yet the mechanism of their connection to long-term health remains obscure. Elucidating the mechanism of the connection is of critical importance because it could reveal a modifiable risk factor, assist in illness detection, and help guide treatment.

Based on a confluence of clinical, neuropsychological, physiological, and genetic evidence, I propose that an inherent hyperexcitability of the neurological system is at the heart of the connection between constitutionally-elevated vital signs and vulnerability to illness. Hypothetically, a failure of the neurological system to self-regulate not only increases the baseline activity of every physiological function, but it also causes a heightened responsivity and slowness to recover that manifests physiologically as a perseveration of the stress response, and cognitive-emotionally as a perseveration of thoughts, feelings, and behaviors [42, 43]. The associated sympathetic overdrive hastens the development and progression of various disease processes $[42,43]$, thus explaining how a subtle elevation in vital signs, which is reflective of that overdrive [27, 49], can be predictive of long-term health $[21,27,30,31,36,40]$. Neuronal hyperexcitability could also explain why severe mental illness, which is known to identify those with the most severe autonomic dysregulation, is associated with such a shortened lifespan [27].

Although the impact of the proposed abnormality, even after adjusting for confounding factors, such as physical fitness, alcohol use, and cigarette smoking, is highly significant, its effects are severely underestimated because most of the so-called "confounding factors" are hypothetically driven by neuronal hyperexcitability. The amplification of stress that is driven by neuronal hyperexcitability can rob affected persons of the motivation to exercise; it can cause them to make poor dietary choices; it can cause them to smoke excessively, drink excessively, and use addictive drugs; it can cause them to be impulsive, contentious, and take unnecessary risks; and it can cause them to become emotionally exhausted, lose hope, and, in extreme cases, take their own lives. The association of these behaviors with neuronal hyperexcitability is supported by the comparatively high frequency with which they occur in persons with psychiatric disorders [45]. When the aforementioned effects are factored in, it becomes apparent that the behavioral consequences of neuronal hyperexcitability can be even more destructive than the abnormality itself and that the external consequences are, in essence, an outward expression of the dysregulation that the abnormality is causing within [42]. This has profound implications because neuronal hyperexcitability is a readily modifiable risk factor. Any habit, food, or chemical that calms the brain could potentially help mitigate the trait of neuronal hyperexcitability [45]. Examples include judicious stress management, maintaining an early sleep schedule, exercising in moderation, enjoying a relaxing hobby, avoiding caffeine and other psychostimulants, minimizing refined sugar, and leaning toward a plant-based diet [45]. Although the benefits of these interventions are widely known, the trait of neuronal hyperexcitability could help explain why some persons fail to implement them, and others remain at high risk even if they do implement them. Hypothetically, such persons, when presenting with upperend-of-normal vital signs, would benefit from treatment with neuroregulators [93] (i.e., anticonvulsants and other braincalming medications).

\section{Conclusion}

The proposed identification of a readily modifiable, easy to detect, predisposing factor for a wide range of mental and physical illnesses has enormous implications in the field of medicine. Recognition of the FLASH trait as the underlying driver of the connection between constitutionally-elevated vital signs and disease risk could give healthcare professionals the ability to better educate high-risk patients and more accurately guide them on how to reduce their risk regardless of their clinical status. FLASH could also provide the first objective, quantifiable, physiological basis for the development of psychiatric symptoms, an advance that would circumvent diagnostic ambiguity, provide a consistent target for treatment, and reduce the stigma of mental illness. Also, by reconceptualizing psychiatric symptoms as the first subjective markers of systemic disease, FLASH could help dispel long-held misconceptions about mental illness, encourage patients to be more forthcoming about their symptoms, and curtail discriminatory practices that limit insurance coverage for mental health services. Finally, the ease of identifying the FLASH trait could prompt otherwise unsuspecting persons to become more aware of their health and seek formal evaluation early on, as it would afford them the ability to assess their own genetic vulnerability as well as 
their progress in treatment.

\section{Disclosure Statement}

The author declares that this article was conceived and written in the absence of any commercial or financial relationships that could be construed as a potential conflict of interest.

\section{References}

[1] Colangelo LA, Yano Y, Jacobs Jr DR, Lloyd-Jones DM. Association of Resting Heart Rate With Blood Pressure and Incident Hypertension Over 30 Years in Black and White Adults: The CARDIA Study. Hypertension 2020; 76 (3): 692698

[2] Shi Y, Zhou W, Liu S, et al. Resting heart rate and the risk of hypertension and heart failure: a dose-response metaanalysis of prospective studies. J Hypertens 2018; 36 (5): 995-1004.

[3] Shen L, Wang Y, Jiang X, et al. Dose-response association of resting heart rate and hypertension in adults: A systematic review and meta-analysis of cohort studies. Medicine (Baltimore) 2020; 99 (10): e19401.

[4] Dalal J, Dasbiswas A, Sathyamurthy I, et al. Heart Rate in Hypertension: Review and Expert Opinion. International Journal of Hypertension 2019; 2019.

[5] Lee DH, de Rezende LFM, Hu FB, Jeon JY, Giovannucci EL. Resting heart rate and risk of type 2 diabetes: a prospective cohort study and meta-analysis. Diabetes Metab Res Rev 2019, 35 (2): e3095.

[6] Aune D, o'Hartaigh B, Vatten LJ. Resting heart rate and the risk of type 2 diabetes: A systematic review and dose-response meta-analysis of cohort studies. Nutr Metab Cardiovasc Dis 2015; 25 (6): 526-534.

[7] Nagaya T, Yoshida H, Takahashi H, Kawai M. Resting heart rate and blood pressure, independent of each other, proportionally raise the risk for type- 2 diabetes mellitus. Int $\mathbf{J}$ Epidemiol 2010; 39 (1): 215-222.

[8] Kannel W, Kannel C, Paffenbarger R, Cupples A. Heart rate and cardiovascular mortality: The Framingham study. Am Heart J 1987; 113: 1489-1494.

[9] Gillum R, Makuc D, Feldman J. Pulse rate, coronary heart disease, and death: The NHANES I epidemiologic follow-up study. Am Heart J 1991; 121: 172-177.

[10] Cooney MT, Vartiainen E, Laatikainen T, et al. Elevated resting heart rate is an independent risk factor for cardiovascular disease in healthy men and women. Am Heart J 2010; 159 (4): 612-619.

[11] Khan H, Kunutsor S, Kalogeropoulos AP, et al. Resting heart rate and risk of incident heart failure: three prospective cohort studies and a systematic meta-analysis. J Am Heart Assoc 2015; 4 (1): e001364.

[12] Alhalabi L, Singleton MJ, Oseni AO, et al. Relation of higher resting heart rate to risk of cardiovascular versus noncardiovascular death. Am J Cardiol 2017; 119 (7): 10031007.
[13] Zhang D, Shen X, Qi X. Resting heart rate and all-cause and cardiovascular mortality in the general population: a metaanalysis. CMAJ 2016; 188 (3): E53-E63.

[14] Yu J, Dai L, Zhao Q. Association of cumulative exposure to resting heart rate with risk of stroke in general population: The Kailuan Cohort Study. Journal of Stroke and Cardiovascular Diseases 2017; (26): 11: 2501-2509.

[15] Huang Y-Q, Shen G, Huang J-Y, Zhang B, Feng Y-Q. A nonlinear association between resting heart rate and ischemic stroke among community elderly hypertensive patients. Postgrad Med 2020; 132 (2): 215-219.

[16] Aune D, Sen A, o'Hartaigh B, et al. Resting heart rate and the risk of cardiovascular disease, total cancer, and all-cause mortality - A systematic review and dose-response metaanalysis of prospective studies. Nutr Metab Cardiovasc Dis 2017; 27 (6): 504-517.

[17] Eriksen BO, Småbrekke S, Jenssen TG, et al. Office and ambulatory heart rate as predictors of age-related kidney function decline: A population-based cohort study. Hypertension 2018; 72 (3): 594-601.

[18] Anker MS, Ebner N, Hildebrandt B, et al. Resting heart rate is an independent predictor of death in patients with colorectal, pancreatic, and non-small cell lung cancer: results of a prospective cardiovascular long-term study. European Journal of Heart Failure 2016; 18 (12).

[19] Park J, Kim JH, Park Y. Resting heart rate is an independent predictor of advanced colorectal adenoma recurrence. PLoS One 2018; 13 (3): e0193753.

[20] Burke SL. Resting heart rate moderates the relationship between neuropsychiatric symptoms, MCI, and Alzheimer's disease. Innov Aging 2019; 3 (suppl 1): S641.

[21] Jouven X, Empana J-P, Schwartz PJ, et al. Heart-rate profile during exercise as a predictor of sudden death. N Engl J Med 2005; 352: 1951-1958.

[22] Latvala A, Kuja-Halkola R, Rick C, et al. Association of resting heart rate and blood pressure in late adolescence with subsequent mental disorders: A longitudinal population study of more than 1 million men in Sweden. JAMA Psychiatry 2016; 73 (12): 1268-1275.

[23] Kemp AH, Brunoni AR, Santos IS, et al. Effects of depression, anxiety, comorbidity, and antidepressants on resting-state heart rate and its variability: an ELSA-Brasil cohort baseline study. Am J Psychiatry 2014; 171 (12): 1328-1334.

[24] Cicek Y, Durakoglugil ME, Kocaman SA, et al. Increased pulse wave velocity in patients with panic disorder: independent vascular influence of panic disorder on arterial stiffness. J Psychosom Res 2012; 73 (2): 145-148.

[25] Paulus EJ, Argo TR, Egge JA. The impact of posttraumatic stress disorder on blood pressure and heart rate in a veteran population. J Trauma Stress 2013; 26 (1): 169-172.

[26] Pole N. The psychophysiology of posttraumatic stress disorder: a meta-analysis. Psychol Bull 2007; 133 (5): 725-746.

[27] Alvares GA, Quintana DS, Hickie IB, Guastella AJ. Autonomic nervous system dysfunction in psychiatric disorders and the impact of psychotropic medications: a systematic review and meta-analysis. J Psychiatry Neurosci 2016; 41 (2): 89-104. 
[28] Carney RM, Freedland KE, Veith RC. Depression, the autonomic nervous system, and coronary heart disease. Psychosom Med 2005; 67 (suppl 1): S29-S33.

[29] Clamor A, Lincoln TM, Thayer JF, Koenig J. Resting vagal activity in schizophrenia: meta-analysis of heart rate variability as a potential endophenotype. Br J Psychiatry 2016; 208 (1): 9-16.

[30] Erlangsen A, Andersen PK, Toender A, et al. Cause-specific life-years lost in people with mental disorders: a nationwide, register-based cohort study. The Lancet 2017; DOI: 10.1016/s2215-0366 (17)30429-7.

[31] Baumert M, Linz D, Stone K, et al. Mean nocturnal respiratory rate predicts cardiovascular and all-cause mortality in community-dwelling older men and women. European Respiratory Journal 2019; DOI: 10.1183/13993003.02175-2018.

[32] Eick C, Groga-Bada P, Reinhardt K, et al. Nocturnal respiratory rate as a predictor of mortality in patients with acute coronary syndrome. Openheart 2018; 5 (2).

[33] Dommasch M, Sinnecker D, Barthel P, et al. Nocturnal respiratory rate predicts non-sudden cardiac death in survivors of acute myocardial infarction. J Am Coll Cardiol 2014; 63: 2432-2433.

[34] Yano Y, Kario K. Nocturnal blood pressure and cardiovascular disease: a review of recent advances. Hypertension Research 2012; 35: 695-701.

[35] Gottesman RF, Schneider ALC, Albert M. Midlife hypertension and 20-year cognitive change: the atherosclerosis risk in communities neurocognitive study. JAMA Neurol 2014; 71 (10): 1218-1227.

[36] Barletta G-M, Flynn J, [...], Furth S, et al. Heart rate and blood pressure variability in children with chronic kidney disease: a report from the CKiD study. Pediatr Nephral 2014; 29 (6): 1059-1065.

[37] Tsuji H, Larson MG, Venditti FJ, et al. Impact of reduced heart rate variability on risk for cardiac events. The Framingham Heart Study. Circulation 1996; 94: 2850-2855.

[38] Thanou A, Stavrakis S, Dyer JW, et al. Impact of heart rate variability, a marker for cardiac health, on lupus disease activity. Arthritis Res Ther 2016; 18 (1): 197.

[39] Cole CR, Blackstone EH, Pashkow FJ, Snader CE, Lauer MS. Heart rate recovery immediately after exercise as a predictor of mortality. N Engl J Med 1999; 341: 1351-1357.

[40] Ó Hartaigh B, Gill TM, [...], Hardy R, et al. Association between resting heart rate across the life course and all-cause mortality: longitudinal findings from the Medical Research Council (MRC) National Survey of Health and Development (NSHD). J Epidemiol Community Health 2014; 68 (9): 883-889.

[41] Sanchez-Conde P, Beltran-Velasco AI, Clemente-Suarez VJ. Influence of psychological profile in autonomic response of nursing students in their first hospital clinical stays. Physiology \& Behavior 2019; 207: 99-103.

[42] McEwen BA. Stress, adaptation, and disease allostasis and allostatic load. Annals of the New York Academy of Sciences 1998; 840 (1): 33-44.

[43] Larsen BA, Christenfeld NJS. Cardiovascular disease and psychiatric comorbidity: the potential role of preservative cognition. Cardiovascular Psychiatry and Neurology 2009;
Article ID: 791017.

[44] Blom EH, Serlachius E, Chesney MA, Olsson EMG. Adolescent girls with emotional disorders have a lower endtidal $\mathrm{CO} 2$ and increased respiratory rate compared with healthy controls. Psychophysiology 2014; 51 (5): 412-418.

[45] Binder MR. The multi-circuit neuronal hyperexcitability hypothesis of psychiatric disorders. AJCEM 2019; 7 (1): 1230 .

[46] Yizhar O, Fenno LE, Prigge M, et al. Neocortical excitation/inhibition balance in information processing and social dysfunction. Nature 2011; 477: 171-178.

[47] Binder MR. Electrophysiology of seizure disorders may hold key to the pathophysiology of psychiatric disorders. AJCEM 2019; 7 (5): 103-110.

[48] Takarae Y, Sweeney J. Neural hyperexcitability in autism spectrum disorders. Brain Sciences 2017; 7 (129).

[49] Narkiewicz K, van de Borne P, Montano N, et al. Sympathetic neural outflow and chemoreflex sensitivity are related to spontaneous breathing rate in normal men. Hypertension 2005; 47 (1): 51-55.

[50] Bradly AJ, Dinan TG. A systematic review of hypothalamicpituitary-adrenal axis function in schizophrenia: implications for mortality. J Psychopharmacol 2010; 24 (4) 91-118.

[51] Bahremand A, Ziai P, Khodadad TK, et al. Agmatine enhances the anticonvulsant effect of lithium chloride on pentylenetetrazole-induced seizures in mice: Involvement of L-arginine/nitric oxide pathway. Epilepsy \& Behavior 2010; 18 (3): 186-192.

[52] Brown P, Kashiviswanath S, Huynh A, et al. Lithium therapy in comorbid temporal lobe epilepsy and cycloid psychosis. Oxf Med Case Reports 2016; (12): omw089.

[53] Shukla S, Mukherjee S, Decina P. Lithium in the treatment of bipolar disorders associated with epilepsy: an open study. J Clin Psychopharmacol 1988; 8 (3): 201-204.

[54] Szabo ST, Blier P. Functional and pharmacological characterization of the modulatory role of serotonin on the firing activity of locus coeruleus norepinephrine neurons. Brain Research 2001; 922 (1): 9-20.

[55] Khedr EM, Elserogy Y, Fawzy M. Effect of psychotropic drugs on cortical excitability of patients with major depressive disorders: A transcranial magnetic stimulation study. Psychiatry Research 2020; 291: 113287.

[56] Ressler KJ, Mayberg HS. Targeting abnormal neural circuits in mood and anxiety disorders: from the laboratory to the clinic. Nat Neurosci 2007; 10 (9): 1116-1124.

[57] El-Mallakh RS, Vöhringer PA, Ostacher MM, et al. Antidepressants worsen rapid-cycling course in bipolar depression: A STEP-BD randomized clinical trial. Journal of Affective Disorders 2015; 184: 318-321.

[58] Goldberg JF, Truman CJ. Antidepressant-induced mania: an overview of current controversies. Bipolar Disorders 2001; 5 (6)

[59] Truman CJ, Goldberg JF, Ghaemi SN. Self-reported history of manic/hypomanic switch associated with antidepressant use: data from the Systematic Treatment Enhancement Program for Bipolar Disorder (STEP-BD). J Clin Psychiatry 2007; 68 (10): 1472-1479. 
[60] Henry C, Sorbara F, Lacoste J, Gindre C, Leboyer M. Antidepressant-induced mania in bipolar patients: Identification of risk factors. J Clin Psychiatry 2001; 62 (4): 249-255.

[61] Yamaguchi Y, Kimoto S, Nagahama T, Kishimoto T. Dosagerelated nature of escitalopram treatment-emergent mania/hypomania: a case series. Neuropsychiatr Dis Treat 2018; 14: 2099-2104.

[62] Licht CM, de Geus EJ, van Dyck R, Penninx BW. Longitudinal evidence for unfavorable effects of antidepressants on heart rate variability. Biol Psychiatry2010; 68 (9): 861-868.

[63] Dawood T, Lambert EA, Barton DA, et al. Specific serotonin reuptake inhibition in major depressive disorder adversely affects novel markers of cardiac risk. Hypertens Res 2007; 30 (4): 285-293.

[64] Kim HJ, Yang J, Lee M-S. Changes of heart rate variability during methylphenidate treatment in attention-deficit hyperactivity disorder children: A 12-week prospective study. Yonsei Med J 2015; 56 (5): 1365-1371.

[65] Koschke M, Boettger MK, Schulz S, et al. Autonomy of autonomic dysfunction in major depression. Psychosom Med 2009; 71 (8): 852-860.

[66] Udupa K, Thirthalli J, Sathyaprabha TN, et al. Differential actions of antidepressant treatments on cardiac autonomic alterations in depression: A prospective comparison. Asian Journal of Psychiatry 2011; 4: 100-106.

[67] Yeragani VK, Roose S, Mallavarapu M, Radhakrishna RK, Pesce V. Major depression with ischemic heart disease: effects of paroxetine and nortriptyline on measures of nonlinearity and chaos of heart rate. Neuropsychobiology 2002; 46: 125-135.

[68] Van Zyl LT, Hazegawa T, Nagata K. Effects of antidepressant treatment on heart rate variability in major depression: A quantitative review. BioPsychSocial Medicine 2008; (2) 12.

[69] Kemp AH, Quintana DS, Quinn CR, Hopkinson P, Harris AW. Major depressive disorder with melancholia displays robust alterations in resting state heart rate and its variability: implications for future morbidity and mortality. Front Psychol 2014; 5: 1387.

[70] Herr RM, Barrech A, Reidel N, et al. Long-term effectiveness of stress management at work: Effects of the changes in perceived stress reactivity on mental health and sleep problems seven years later. Int J Environ Res Public Health 2018; 15 (2): 255.

[71] Brainstorm Consortium; Anttila V, Bulik-Sullivan B, Finucane HK, et al. Analysis of shared heritability in common disorders of the brain. Science 2018; 360 (6395): eaap8757.

[72] Beissner F, Meissner K, Bär KJ, Napadow V. The autonomic brain: an activation likelihood estimation meta-analysis for central processing of autonomic function. J Neurosci 2013; 33 (25): 10503-10511.

[73] Keyes KM, Eaton NR, Hasin DS. Childhood maltreatment and the structure of common psychiatric disorders. British Journal of Psychiatry 2012; 200 (2): 107-115.

[74] Uher R, Zwicker A. Etiology in psychiatry: embracing the reality of poly-gene-environmental causation of mental illness. World Psychiatry 2017; 16 (2).
[75] Rosenman RH, Brand RJ, Sholtz RI, Friedman M. Multivariate prediction of coronary heart disease during 8.5 year follow-up in the Western Collaborative Group study. The American Journal of Cardiology 1976; 37 (6): 903-910.

[76] Kupper N, Donollet J. Type D personality as a risk factor in coronary heart disease: a review of current evidence. Curr Cardiol Rep 2018; 20 (11): 104.

[77] Ferreira, MAR, O’Donovan MC, [...], Sklar P. Collaborative genome-wide association analysis supports a role for ANK3 and CACNA1C in bipolar disorder. Nat Genet 2008; 40 (9): 1056-1058.

[78] Lopez AY, Wang $\mathrm{X}, \mathrm{Xu} \mathrm{M}$, et al. Ankyrin-G isoform imbalance and interneuronopathy link epilepsy and bipolar disorder. Mol Psychiatry 2017; 22 (10): 1464-1472.

[79] Green EK, Grozeva D, Jones I, et al., Wellcome Trust Case Control Consortium, Holmans, PA, Owen, MJ, O'Donovan, MC Craddock N. The bipolar disorder risk allele at CACNA1C also confers risk of recurrent major depression and of schizophrenia. Mol Psychiatry 2010; 15 (10): 1016-1022.

[80] Liu Y, Blackwood DH, Caesar S, et al. Meta-analysis of genome-wide association data of bipolar disorder and major depressive disorder. Mol Psychiatry 2011; 16 (1).

[81] Iqbal Z, Vandeweyer G, van der Voet M, et al. Homozygous and heterozygous disruptions of ANK3: at the crossroads of neurodevelopmental and psychiatric disorders. Human Molecular Genetics 2013; 22: 1960-1970.

[82] Santos M, D'Amico D, Spadoni O, et al. Hippocampal hyperexcitability underlies enhanced fear memories in TgNTRK3, a panic disorder mouse model. Journal of Neuroscience 2013; 33 (38): 15259-15271.

[83] Contractor A, Klyachko VA, Portera-Cailliau C. Altered neuronal and circuit excitability in Fragile $\mathrm{X}$ syndrome. Neuron 2015; 87 (4): 699-715.

[84] O'Brien NL, Way MJ, Kandaswamy R, et al. The functional GRM3 Kozak sequence variant rs148754219 affects the risk of schizophrenia and alcohol dependence as well as bipolar disorder. Psychiatric Genetics 2014; 24: 277-278.

[85] Schizophrenia Working Group of the Psychiatric Genomics Consortium: Ripke S, Neale BM, [...], O'Donovan MC. Biological insights from 108 schizophrenia-associated genetic loci. Nature 2014; 511 (7510): 421-427.

[86] Freedman R, Coon H, Myles-Worsley M, et al. Linkage of a neurophysiological deficit in schizophrenia to a chromosome 15 locus. PNAS 1997; 94 (2): 587-592.

[87] Andrade A, Brennecke A, Mallat S, et al. Genetic associations between voltage-gated calcium channels and psychiatric disorders. Int J Mol Sci 2019; 20 (14): 3537.

[88] Kim J-B. Channelopathies. Korean J of Pediatr 2014; 57 (1): $1-18$.

[89] Brunklaus A, Ellis R, Reavey E, Semsarian C, Zuberi SM. Genotype phenotype associations across the voltage-gated sodium channel family. J Med Genet 2014; 51 (10): 650-658.

[90] D'Agnelli S, Arendt-Nielsen L, Gerra MC, et al. Fibromyalgia: Genetics and epigenetics insights may provide the basis for the development of diagnostic biomarkers. Molecular Pain $2019 ; 15$. 
[91] Van de Borne P, Montano N, Zimmerman B, Pagani M, Somers VK. Relationship between repeated measures of hemodynamics, muscle sympathetic nerve activity, and their spectral oscillations. Circulation 1997; 96: 4326-4332.

[92] Grunze HCR. The effectiveness of anticonvulsants in psychiatric disorders. Dialogues Clin Neurosci 2008; 10 (1): 77-89.
[93] Binder MR. Introducing the term "Neuroregulator" in Psychiatry. AJCEM 2019; 7 (3): 66-70.

[94] Hallioglu O, Okuyaz C, Mert E, Makharoblidze K. Effects of antiepileptic drug therapy on heart rate variability in children with epilepsy. Epilepsy Res 2008; 79 (1): 49-54 